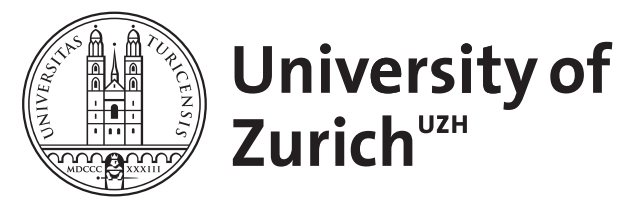

\title{
Hepatitis-B- und -C-assoziierte Glomerulonephritiden
}

\author{
Fehr, T ; Gaspert, A ; Varga, Z ; Bosshard, A ; Ambühl, P M
}

\begin{abstract}
Virale Hepatitiden sind häufig mit extrahepatischen Manifestationen assoziiert. Bei der HepatitisB ist die membranöse Glomerulonephritis (GN) die häufigste histologische Diagnose. Im Rahmen der HepatitisC wird vorwiegend eine membranoproliferative GN mit oder ohne gemischte Kryoglobulinämie beobachtet. Eine zentrale pathogenetische Rolle spielen Immunkomplexe (virale Antigene, antivirale Antikörper, bei Kryoglobulinämie auch Rheumafaktoren). Diese Komplexe werden in der Niere abgelagert und aktivieren Komplement, was schließlich zum Nierenschaden führt. Therapeutisch zentral ist die antivirale Therapie mit dem Ziel der Antigenelimination. Im Falle der HepatitisB kann eine Therapie mit IFN durchgeführt werden, alternativ mit Lamivudin. Eine immunsuppressive Therapie steht eher im Hintergrund. Bei der HepatitisC ist die Standardtherapie IFN in Kombination mit Ribavirin. Bei einer zusätzlichen Kryoglobulinämie besteht die Alternative einer Therapie mit Rituximab, bei schwerem Verlauf mit Plasmapherese, Steroiden und Cyclophosphamid. Bei vollständiger Elimination der Virusreplikation ist die Prognose dieser sekundären GN günstig
\end{abstract}

DOI: https://doi.org/10.1007/s11560-006-0054-y

Posted at the Zurich Open Repository and Archive, University of Zurich ZORA URL: https://doi.org/10.5167/uzh-156232

Journal Article

Published Version

Originally published at:

Fehr, T; Gaspert, A; Varga, Z; Bosshard, A; Ambühl, P M (2007). Hepatitis-B- und -C-assoziierte Glomerulonephritiden. Der Nephrologe, 2(1):37-43.

DOI: https://doi.org/10.1007/s11560-006-0054-y 
Nephrologe 2007 $\cdot 2: 37-43$ DOI 10.1007/s11560-006-0054-y Online publiziert: 22. Dezember 2006

(c) Springer Medizin Verlag 2006

Redaktion

R.P. Wüthrich, Zürich
T. Fehr ${ }^{1} \cdot$ A. Gaspert ${ }^{2}$. Z. Varga ${ }^{2}$ - A. Bosshard ${ }^{1}$ P. M. Ambühl ${ }^{1}$

${ }^{1}$ Klinik für Nephrologie, Universitätsspital Zürich

${ }^{2}$ Departement Pathologie, Universitätsspital Zürich

\section{Hepatitis-B- und -C-assoziierte Glomerulonephritiden}

\begin{abstract}
Virale Hepatitiden, speziell die Hepatitis B und $C$ im chronischen Verlauf, sind gehäuft mit extrahepatischen Manifestationen assoziiert [3], die unabhängig von der Grundkrankheit zu erheblicher Morbidität und auch Mortalität beitragen können. Unterschieden werden klinisch symptomatische von rein serologischen (Nachweis von Autoantikörpern) extrahepatischen Manifestationen. Die absolute Häufigkeit ist von Studie zu Studie unterschiedlich, ist aber allgemein bei der Hepatitis $C$ deutlich höher als bei der Hepatitis B (• Tab. 1). Unter den verschiedenen beteiligten Organen steht die Niere meist im Vordergrund. Im Rahmen der Hepatitis B ist die membranöse Glomerulonephritis (GN) mit nephrotischem Syndrom die häufigste histologisch gesicherte Diagnose, gefolgt von der membranoproliferativen GN und der Panarteriitis nodosa [5, 15]. Im Gegensatz dazu wird im Rahmen der Hepatitis $\mathrm{C}$ vorwiegend eine membranoproliferative GN mit oder ohne gleichzeitig bestehende gemischte Kryoglobulinämie und begleitendem nephritischem Syndrom diagnostiziert [24]. Eine IgA-Nephropathie kann bei Hepatitis $B$ und $C$ ebenfalls vorkommen.
\end{abstract}

\section{Pathogenese der renalen Beteiligung}

Die Pathogenese der hepatitisassoziierten Glomerulonephritiden ist noch nicht definitiv geklärt. Doch sowohl bei der Hepatitis $B$ als auch bei der Hepatitis C scheinen zirkulierende Immunkomplexe, die in der Niere deponiert werden und dort
Entzündungsreaktionen mit Komplementaktivierung auslösen, involviert zu sein $[5,22]$. Die gleichzeitige chronische Präsenz von Antigen und Antikörpern im Serum begünstigt die Immunkomplexbildung [13], und sorgfältige Studien haben die Assoziation mit der Virämie dokumentiert. Im Rahmen der Hepatitis B wurde mittels immunhistologischen Untersuchungen die Ablagerung von $\mathrm{HBs} \mathrm{Ag}$, $\mathrm{HBcAg}$ und $\mathrm{HBeAg}$ in der glomerulären Basalmembran bei Patienten mit membranöser GN gezeigt (Review in [5]). Eine weitere Studie hat bei 41 von 43 Patienten (95\%) mittels In-situ-Hybridisierung auch HBV-DNA nachgewiesen [16]. Ähnliche Studien liegen für die membranoproliferative GN im Rahmen der Hepatitis C vor, besonders wenn sie mit einer gemischten Kryoglobulinämie assoziiert ist.

\section{- Bei der gemischten Kryoglobulinämie zirkulieren Komplexe von Rheumafaktoren (IgM-Anti-lgG- Antikörper) und IgG im Serum.}

Die gemischten Kryoglobulinämien können als Typ II (monoklonaler Rheumafaktor und polyklonales IgG) und als Typ III (Rheumafaktor und IgG polyklonal) klassifiziert werden. Kryoglobuline im Kontext einer HCV-Infektion enthalten $\mathrm{zu}$ über 90\% Anti-HCV-Antikörper. Sorgfältige Analysen haben in diesen Komplexen HCV-Virionen (Antigen), Anti-HCV-Antikörper (IgG) und Rheumafaktoren nachgewiesen $[1,28,30]$. Experimentelle Modelle suggerieren, dass die repetitive Struktur von Virus-Anti-VirusIgG-Komplexen die Aktivierung von BZellen und somit die Synthese von Rheumafaktoren begünstigt [12].
Neben Immunkomplexen dürften auch noch andere Mechanismen in der Pathogenese einer hepatitisassoziierten GN eine Rolle spielen, so zum Beispiel ein direkter zytopathischer Effekt der Hepatitisviren in der Niere oder virusinduzierte zelluläre und/oder humorale Immuneffektormechanismen (T-Zellen, Antikörper, Zytokine), die das Nierengewebe schädigen könnten [5]. Ein Beitrag solcher Mechanismen ist jedoch im Gegensatz zur Immunkomplexablagerung noch wenig gut dokumentiert. Speziell der Nachweis von viraler Replikation im extrahepatischem Gewebe, welche die Grundlage eines direkt zytopathischen Effektes wäre, hat sich als methodologisch sehr schwierig erwiesen [7].

In der Folge möchten wir anhand zweier Fallberichte das aktuelle Management von Patienten mit Hepatitis-B- und -C-assoziierten Glomerulonephritiden diskutieren.

\section{HBV-assoziierte membranöse GN}

\section{Fallvignette}

Ein 46-jähriger Patient (P.W.) wurde uns wegen ausgedehnter Beinödeme, Aszites und einer Gewichtszunahme von $19 \mathrm{~kg}$ zur weiteren Abklärung zugewiesen. Die Vorgeschichte des Patienten war mit Ausnahme einer Inguinalhernienoperation vor 8 Jahren unauffällig. In der Familienanamnese zeigte sich eine mütterlicherseits vererbte Zystennierenerkrankung, von der der Patient nicht betroffen ist. Die internistische Diagnostik ergab als mögliche Erklärung für den Aszites eine Leberzirrhose auf dem Boden einer replizierenden Hepatitis-B-Infektion (initiale Virämie 110.00o.00o IE/ml). In der Le- 


\begin{tabular}{|c|c|c|}
\hline & Chronische Hepatitis B $[3,9]$ & Chronische Hepatitis C $[21,24]$ \\
\hline \multirow[t]{3}{*}{ Häufigkeit } & Klinisch: $16 \%$ & Klinisch: 38\% \\
\hline & Serologisch: $15 \%$ & Serologisch: $74 \%$ \\
\hline & Kinder: häufig & Kinder: selten \\
\hline \multirow[t]{2}{*}{ Renal } & $\begin{array}{l}\text { Häufig/typisch: membranöse } \\
\text { Glomerulonephritis }\end{array}$ & $\begin{array}{l}\text { Häufig/typisch: membranoproliferative Glo- } \\
\text { merulonephritis mit/ohne Kryoglobulinämie }\end{array}$ \\
\hline & $\begin{array}{l}\text { Selten: Panarteriitis nodosa } \\
\text { Membranoproliferative Glomerulo- } \\
\text { nephritis } \\
\text { Mesangial-proliferative Glomerulo- } \\
\text { nephritis einschließlich IgA-Nephro- } \\
\text { pathie }\end{array}$ & $\begin{array}{l}\text { Selten: membranöse Glomerulonephritis } \\
\text { Fibrilläre Glomerulonephritis } \\
\text { IgA-Nephropathie }\end{array}$ \\
\hline Nichtrenal & $\begin{array}{l}\text { Panarteriitis nodosa } \\
\text { Thrombozytopenie } \\
\text { Periphere Neuropathie } \\
\text { Sicca-Syndrom } \\
\text { Myalgien/Arthralgien }\end{array}$ & $\begin{array}{l}\text { Gemischte Kryoglobulinämie } \\
\text { Thrombozytopenie } \\
\text { Periphere Neuropathie } \\
\text { Lymphozytäre Sialadenitis } \\
\text { Porphyria cutanea tarda } \\
\text { B-Zell-Non-Hodgkin-Lymphom } \\
\text { Hypothyreose und Diabetes mellitus }\end{array}$ \\
\hline
\end{tabular}

\begin{tabular}{|c|c|c|}
\hline & $\begin{array}{l}\text { HBV-assoziierte membranöse } \\
\text { GN [5] }\end{array}$ & $\begin{array}{l}\text { HCV-assoziierte kryoglobulinämische } \\
\text { membranoproliferative GN [18] }\end{array}$ \\
\hline $\begin{array}{l}\text { First-line- } \\
\text { Therapie }\end{array}$ & PegIFNa (180 ug 1-mal pro Woche) & $\begin{array}{l}\text { PegIFNa }(180 \mu \mathrm{g} 1 \text {-mal pro Woche })+ \\
\text { Ribavirin }(800-1200 \mathrm{mg} / \mathrm{Tag} \text {, dosisadaptiert } \\
\text { an Kreatininclearance) }\end{array}$ \\
\hline $\begin{array}{l}\text { Second-line- } \\
\text { Therapie }^{\mathrm{a}}\end{array}$ & $\begin{array}{l}\text { Lamivudin (100 mg/Tag) } \\
\text { Andere antivirale Substanzen } \\
\text { (Adefovir, Telbivudin)c } \\
\text { Immunsuppression (Prednison } \\
\text { u. a.) nur in Ausnahmefällen und } \\
\text { nur in Kombination mit antiviraler } \\
\text { Therapie }\end{array}$ & $\begin{array}{l}\text { Rituximab ( } 375 \mathrm{mg} / \mathrm{m}^{2} 1 \text {-mal pro Woche für } \\
\text { 2-6 Wochen) } \\
\text { Plasmapherese + Prednison + Rituximab } \\
\text { oder Cyclophosphamid }^{b}\end{array}$ \\
\hline $\begin{array}{l}\text { Allgemeine } \\
\text { Maßnahmen }\end{array}$ & \multicolumn{2}{|c|}{$\begin{array}{l}\text { Antihypertensive und antiproteinurische Therapie (ACE-Hemmer, } \text { AT }_{2} \text {-Rezeptorblo- } \\
\text { cker, Diuretika etc.) }\end{array}$} \\
\hline & \multicolumn{2}{|c|}{$\begin{array}{l}\text { Therapie der Komplikationen des nephrotischen Syndroms (Hyperlipidämie, } \\
\text { Hyperkoagulabilität, Hypogammaglobulinämie) }\end{array}$} \\
\hline \multicolumn{3}{|c|}{$\begin{array}{l}\text { aBei Kontraindikation, Unverträglichkeit oder Therapieversagen der First-line-Therapie. }{ }^{\text {bei sys- }} \\
\text { temischer Vaskulitis, schwerem nephrotischem Syndrom, rasch progredientem Nierenversagen. } \\
\text { Rituximab hat gegenüber Cyclophosphamid den Vorteil, dass es nicht zu einem Anstieg der HCV- } \\
\text { Replikation führt.'Es liegen noch keine Daten dazu vor. }\end{array}$} \\
\hline
\end{tabular}

berbiopsie zeigte sich eine chronische Hepatitis mit geringer Aktivität, eine Portalfibrose (Ishak Score 3) und der Nachweis von HBV-Antigen, passend zu einer chronischen Hepatitis B.

Als Ursache der ausgedehnten Beinödeme und des Aszites wurde ein nephrotisches Syndrom (maximale Proteinurie im Verlauf $10 \mathrm{~g} / 24 \mathrm{~h}$, normale Nierenfunktion mit Kreatininclearance von $130 \mathrm{ml} / \mathrm{min}$ nach Cockcroft) mit Hypalbuminämie und Hyperlipidämie gefunden. Es wurde in der Folge eine Nierenbiopsie durchgeführt, deren Befunde in - Abb. 1 gezeigt sind. Lichtmikroskopisch fand sich eine nur diskret verdick- chronischen HBV-Infektion. Was ist das optimale Management?

\section{Therapie und Prognose}

Die Hepatitis-B-assoziierte membranöse GN kommt bei Kindern und Erwachsenen vor. Je jünger der Patient ist, desto besser sind die Chancen für eine Spontanremission. Bei Kindern kommt es bei $64 \% \mathrm{zu}$ einer Spontanremission, und in über 95\% der Fälle bleibt die Nierenfunktion erhalten [14]. Bei Erwachsenen führt die Krankheit in bis zu $25 \%$ zu einem Nierenversagen im Endstadium (ESRD) [5]. Daraus leitet sich auch die Therapienotwendigkeit ab. Als Option steht grundsätzlich eine antivirale Therapie mit IFNa oder Lamivudin zur Verfügung.

Unspezifische Immunsuppression. Die unspezifische Immunsuppression mit Kortikosteroiden mit bzw. ohne Cyclophosphamid hat in klinischen Studien keinen Erfolg gezeigt. Lai et al. [19] haben 8 Patienten mit HBV-assoziierter GN für $6 \mathrm{Mo}$ nate mit Kortikosteroiden behandelt und mit 7 historischen Kontrollpatienten verglichen, die nur symptomatisch behandelt wurden. Drei von 8 Patienten in der Therapiegruppe und 2 von 7 in der Kontrollgruppe erreichten eine Vollremission des nephrotischen Syndroms. Die Patienten in der Therapiegruppe zeigten jedoch einen Anstieg der Virämie und des $\mathrm{HBeAg}$ im Serum, und 3 von 8 hatten eine transiente Leberdysfunktion (o von 7 in der Kontrollegruppe). Ein ähnlich negatives Resultat wurde berichtet von 2 Patienten, deren HBV-assoziierte membranöse GN mit Mycophenolatmofetil behandelt wurde, auch bei ihnen stieg die Virusreplikation an [29]. Von einer unspezifisch immunsuppressiven Therapie wird wegen fehlender Wirkung, aber gleichzeitigem Risiko von erhöhter Virusreplikation und verzögerter Serokonversion abgeraten.

Antivirale Therapie. Die antivirale Therapie mit Interferon (IFN) oder Lamivudin blockiert die Virusreplikation und entfernt somit die chronische Antigenexposition, welche für die Bildung von Immunkomplexen verantwortlich ist.

Lin et al. [20] behandelten 20 Patienten mit HBV-assoziierter membranöser GN 
mit IFNa und verglichen sie mit 20 nur symptomatisch behandelten Patienten: In der Therapiegruppe waren nach 3 Monaten $100 \%$ der Patienten frei von Proteinurie, während in der Kontrollgruppe 50\% noch immer eine nephrotische und 50\% eine subnephrotische Proteinurie aufwiesen. In der Therapiegruppe erreichten 12 von 20 Patienten eine Serokonversion zu Anti-HBe nach 12 Monaten, in der Kontrollgruppe kein Patient. Ähnlich gute Resultate wurden bei einer Gruppe von schwarzen Kindern in Südafrika erreicht [6]. Damit kann die IFN-Therapie als Goldstandard für diese Krankheit gelten.

\section{(7) Die IFN-Therapie kann als Goldstandard für diese Krankheit gelten}

Als Alternative steht Lamivudin für die antivirale Therapie der Hepatitis B zur Verfügung. Zur Behandlung der HBV-assoziierten membranösen GN liegen jedoch nur einige Fallberichte sowie eine kleine kontrollierte klinische Studie vor. Tang et al. [31] behandelten $10 \mathrm{HBsAg}$-positive Patienten mit bioptisch erwiesener membranöser GN mit Lamivudin und verglichen sie mit 12 historischen Kontrollpatienten, die lediglich eine symptomatische Behandlung erhielten hatten. Die Rate an Vollremissionen bezüglich der Proteinurie in der Therapiegruppe war 4 von 10 nach 6 und 6 von 10 nach 12 Monaten. Die entsprechenden Zahlen der Kontrollgruppe waren 1 von 12 und 3 von 12. Hinsichtlich des Kriteriums „Freiheit von ESRD“ nach 3 Jahren war das kumulative Ergebnis in der Therapiegruppe $100 \%$, in der Kontrollgruppe $58 \%$. Dieses Resultat zeigt eindeutig die günstige Wirkung von Lamivudin, obwohl die Substanz (bei fehlendem Direktvergleich) etwas weniger wirksam scheint als IFNa.

$\mathrm{Zu}$ neueren antiviralen Substanzen wie Adefovir oder Telbivudin liegen keine Daten für diese Krankheit vor.

\section{HCV-assoziierte membra- noproliferative GN mit und ohne Kryoglobulinämie}

\section{Fallvignette}

Eine 54-jährige Patientin (E.K.) wurde uns zur Abklärung eines subakut aufge-

Nephrologe 2007 · 2:37-43 DOI 10.1007/s11560-006-0054-y

(c) Springer Medizin Verlag 2006

T. Fehr · A. Gaspert · Z. Varga · A. Bosshard · P. M. Ambühl Hepatitis-B- und -C-assoziierte Glomerulonephritiden

\section{Zusammenfassung}

Virale Hepatitiden sind häufig mit extrahepatischen Manifestationen assoziiert. Bei der Hepatitis B ist die membranöse Glomerulonephritis (GN) die häufigste histologische Diagnose. Im Rahmen der Hepatitis C wird vorwiegend eine membranoproliferative GN mit oder ohne gemischte Kryoglobulinämie beobachtet. Eine zentrale pathogenetische Rolle spielen Immunkomplexe (virale Antigene, antivirale Antikörper, bei Kryoglobulinämie auch Rheumafaktoren). Diese Komplexe werden in der Niere abgelagert und aktivieren Komplement, was schließlich zum Nierenschaden führt. Therapeutisch zentral ist die antivirale Therapie mit dem Ziel der Antigenelimination. Im Falle der Hepatitis B kann ei-

\section{Hepatitis B and C associated glomerulonephritides}

\section{Abstract}

Viral hepatitis is often associated with extrahepatic manifestations, especially in the kidney. In the context of hepatitis B, membranous nephropathy is the most frequent diagnosis, whereas hepatitis $C$ mainly causes membranoproliferative glomerulonephritis with or without cryoglobulinemia. In both cases, immune complexes containing viral antigens, antiviral antibodies and, in the case of cryoglobulinemia, also rheumatoid factors play a critical role in pathogenesis. They are deposited in the kidney and activate complement, which eventually leads to renal injury. Therefore, antiviral treatment is the most important approach having the goal of anti- ne Therapie mit IFNa durchgeführt werden, alternativ mit Lamivudin. Eine immunsuppressive Therapie steht eher im Hintergrund. Bei der Hepatitis $C$ ist die Standardtherapie IFNa in Kombination mit Ribavirin. Bei einer zusätzlichen Kryoglobulinämie besteht die Alternative einer Therapie mit Rituximab, bei schwerem Verlauf mit Plasmapherese, Steroiden und Cyclophosphamid. Bei vollständiger Elimination der Virusreplikation ist die Prognose dieser sekundären GN günstig.

\section{Schlüsselwörter}

Hepatitis B · Hepatitis C · Glomerulonephritis . Kryoglobulinämie $\cdot$ Interferon gen elimination. For hepatitis B, IFNa or alternatively lamivudine have been used. Immunosuppressive therapy is rarely needed. Hepatitis $C$ is treated with IFNa and ribavirin. If cryoglobulinemia is present, rituximab can be used. In severe cases plasmapheresis, steroids and cyclophosphamide may be necessary. If antiviral therapy is successful with the complete elimination of viral replication, the prognosis of these secondary forms of glomerulonephritis is favourable.

\section{Keywords}

Hepatitis B · Hepatitis C · Glomerulonephritis Cryoglobulinemia $\cdot$ Interferon 


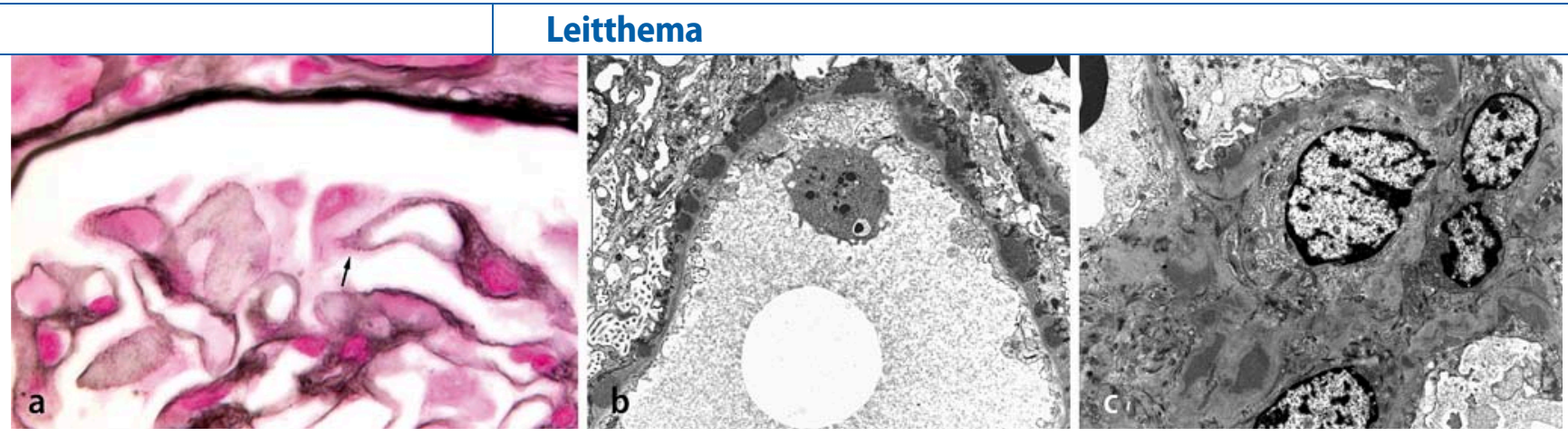

Abb. 1 ABV-assoziierte membranöse GN. a Lichtmikroskopie: Detailaufnahme der glomerulären Basalmembran, die an der äußeren Oberfläche diskret irregulär ist, einzelne "Löcher" aufweist und einzelne sog. Spikes (Pfeil). (Silbermethenamin, Originalvergr. 400:1). b Elektronenmikroskopie: Unregelmäßig verteilte, unterschiedlich große subepitheliale elektronendichte Depots, teils mit benachbarter Basalmembranreaktion (Originalvergr. 4600:1). c Elektronenmikroskopie: subepitheliale und mesangiale elektronendichte Depots (Originalvergr. 4600:1)
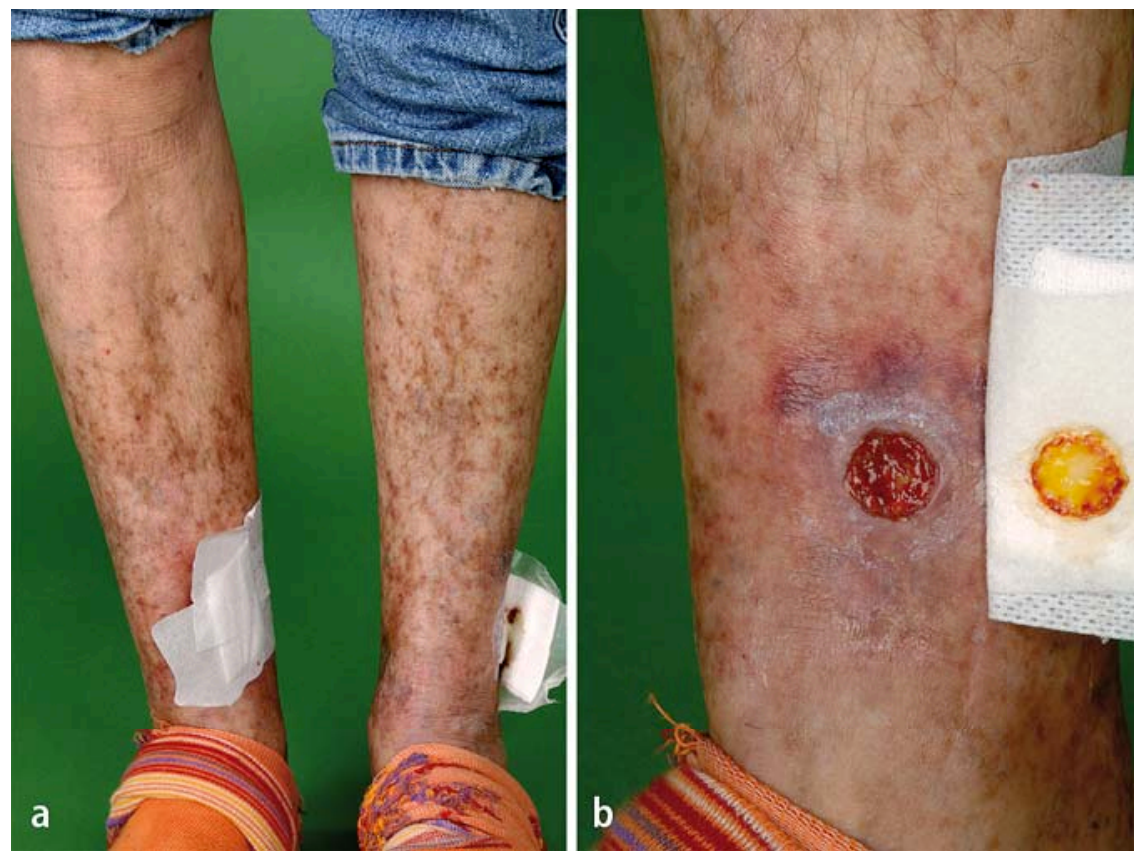

Abb. $2 \Delta$ Unterschenkelulzera einer Patientin mit HCV-assoziierter membranoproliferativer GN und gemischter Kryoglobulinämie

tretenen nephritischen Syndroms zugewiesen. Im Rahmen einer ophthalmologischen Untersuchung wegen eines beidseitigen Papillenödems war ein kombiniert nephritisches und nephrotisches Syndrom entdeckt worden. Die Patientin wies eine Hypertonie (172/84 mmHg) auf. Es wurde ein aktives Sediment mit Erythrozyturie (mit 30\% dysmorphen Formen) und Proteinurie $(8 \mathrm{~g} / 24 \mathrm{~h}$ ) festgestellt. Sonographisch waren die Nieren beidseits unauffällig. Die Nierenfunktion war mit einer Kreatininclearance von $85 \mathrm{ml} / \mathrm{min}$ nach Cockcroft leicht eingeschränkt.

Der Vorgeschichte entnahmen wir einen früheren i.v. Drogenabusus. In diesem Zusammenhang hatte sie sich eine HBVund eine HCV-Infektion zugezogen.
Im Status fielen neben der Hypertonie ausgestanzte Knöchelulzera beidseits und eine Hyperpigmentierung im Unterschenkelbereich auf (• Abb. 2).

Die virologische Diagnostik ergab einen Status nach Hepatitis B (HBsAg und HBV-DNA negativ, Anti-HBc-IgG positiv) und eine aktiv replizierende Hepatitis C (viral load 8.800.00o IE/ml). Es wurde deshalb eine kombinierte transjuguläre Leber- und Nierenbiopsie veranlasst, die folgende Befunde ergab. In der Leber eine chronische Hepatitis mit minimaler Aktivität und geringer Portalfibrose (Ishak Score 1) sowie eine gemischt-tropfige Steatose, aber keine nekroinflammatorischen Läsionen. Die Nierenbiopsie ist in Abb. 3 dargestellt. Die Lichtmik- roskopie zeigte eine endokapillare Proliferation mit einem lobulierten Bild sowie Doppelkonturen der Basalmembran (- Abb. 3a). Zusätzlich kamen intrakapillare hyaline Thromben zur Darstellung. In der Immunfluoreszenz wurden IgG-Ablagerungen gefunden, und die ultrastrukturelle Untersuchung zeigte subendotheliale elektronendichte Depots mit einer retikulären Substruktur.

Abschließend lässt sich somit die Diagnose einer membranoproliferativen GN Typ I mit intrakapillaren hyalinen Thromben bei replizierender chronischer Hepatitis $\mathrm{C}$ stellen. Die intrakapillaren Thromben sind typisch für eine gemischte Kryoglobulinämie, die auch laborchemisch nachgewiesen werden konnte $(19,9 \mathrm{~g} / \mathrm{l})$ und die Ulzera der Patientin erklärt. Wie soll diese Patientin behandelt werden?

\section{Therapie und Prognose}

Wie eingangs erwähnt, spielt auch bei der HCV-assoziierten membranoproliferativen GN die persistierende Virusreplikation eine wichtige Rolle, und somit ist die antivirale Therapie wie bei der HBV-assoziierten GN ein wichtiges Standbein. Ohne Therapie ist die Prognose schlecht. Etwa $25 \%$ der Patienten erleiden einen raschen Abfall der Nierenfunktion, und weitere $50 \%$ haben mittel- bis langfristig eine leicht bis mittelschwer eingeschränkte Nierenfunktion [10]. Die Erfolge der antiviralen Therapie sind jedoch weniger gut als bei der HBV-assoziierten GN - insbesondere bei Vorliegen einer gemischten Kryoglobulinämie - und Rückfälle sind nach Absetzen sehr häufig. Aus diesem Grunde kommt der immunsuppressiven Therapie größere Bedeutung zu. 
Antivirale Therapie. Die zwei ersten Studien zu IFNa haben ein besseres Outcome bezüglich der Nierenfunktion bei Patienten gezeigt, die virologisch angesprochen haben $[17,23]$. In beiden Studien wurde jedoch eine hohe Rückfallrate nach Absetzen der Therapie beobachtet. In den letzten drei Jahren sind nun aber 3 Studien mit insgesamt 28 Patienten sowie einige Fallberichte publiziert worden, in denen eine kombinierte Therapie von IFN (standard oder pegyliert) und Ribavirin verwendet wurde, und deren Resultate bezüglich Rezidiven deutlich besser sind [2, 8, 27]. Die größte Studie (Alric et al. [2]) hat 25 Patienten mit kryoglobulinämischer membranoproliferativer $\mathrm{GN}$ untersucht: Alle erhielten zuerst eine Behandlung mit Prednison, Diuretika oder Plasmapherese. Bei 18 wurde dann eine Therapie mit pegyliertem IFN und Ribavirin durchgeführt (im Mittel für $18 \mathrm{Mo}$ nate mit 6 Monaten Follow-up), 7 Patienten dienten als Kontrollgruppe. Zwölf der 18 behandelten Patienten waren anhaltende virologische Responder.

\section{Derzeit gilt die antivirale Therapie als Standard}

Bei diesen Respondern kam es zu einem signifikanten Abfall der Proteinurie und der Kryoglobulinämie, während Nonresponder und Kontrollen unverändert blieben. Keine Unterschiede zwischen den Gruppen gab es bezüglich Nierenfunktion während der relativ kurzen Beobachtungsdauer. Basierend auf den genannten 3 Studien gilt die antivirale Therapie momentan als Standardtherapie dieser Krankheit.

Immunsuppressive Therapie. Unterschiedliche Kombinationen von Steroiden, Cyclophosphamid und Plasmapherese zur Entfernung der Kryoglobuline im Kontext der HCV-assoziierten Kryoglobulinämie werden kontrovers diskutiert. Wenn sie nicht mit antiviraler Therapie kombiniert ist, kann eine solche Behandlung zwar die Komplikationen der Kryoglobulinämie verbessern, birgt aber gleichzeitig das Risiko eines Aufflammens der HCV-Infektion und einer Verschlechterung der HCV-assoziierten Lebererkrankung. Sie hat ihren Platz deshalb

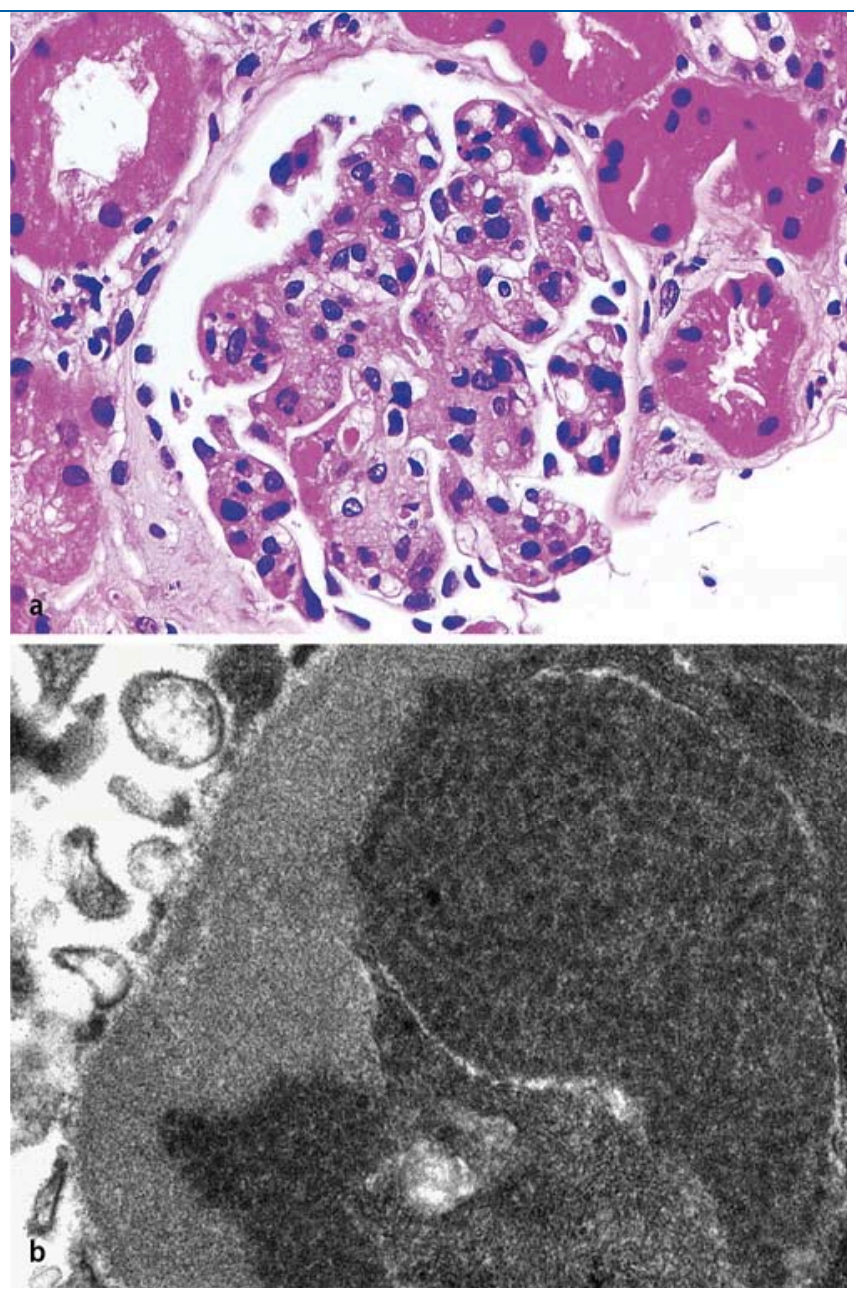

in erster Linie bei schweren akuten Verläufen einer Kryoglobulinämie mit systemischer Vaskulitis und sollte später wenn möglich mit einer antiviralen Therapie kombiniert werden $[11,18]$.

Als neue Alternative zur Behandlung der kryoglobulinämischen membranoproliferativen GN wurde kürzlich Rituximab vorgeschlagen. Rituximab ist ein monoklonaler Anti-CD2o-Antikörper, der B-Zellen selektiv depletiert. Dies ist ein interessanter Ansatz zur Kontrolle der Kryoglobulinproduktion. Drei Studien mit 2, 6 und 5 Patienten mit kryoglobulinämischer membranoproliferativer GN wurden publiziert $[25,26,32]$. In allen wurde Rituximab als Monotherapie eingesetzt. Bei 12 von 13 Patienten wurde ein Rückgang der Proteinurie festgestellt, ebenfalls bei 12 von 13 blieb das Kreatinin stabil oder verringerte sich. Es wurden keine Hinweise auf eine erhöhte Virusreplikation gefunden. Schließlich wurde kürzlich eine Studie mit 7 nierentrans- plantierten Patienten mit kryoglobulinämischer membranoproliferativer GN im Transplantat vorgestellt, die mit Rituximab behandelt wurden [4]. Bei allen Patienten wurde ein deutliches Ansprechen bezüglich Proteinurie und ein praktisches Verschwinden der Kryoglobuline beobachtet. Allerdings erlitten 2 Patienten schwere infektiöse Komplikationen (eine letale disseminierte Kryptokokkose, eine disseminierte HSV2-Infektion). Rituximab ist somit eine interessante Alternative zu Interferon bei Kontraindikationen oder Unverträglichkeit, muss aber im Kontext systemischer Immunsuppression mit Vorsicht eingesetzt werden.

\section{Fazit für die Praxis}

Die Therapieoptionen für die HBV-assoziierte membranöse und die HCV-assoziierte kryoglobulinämische membranoproliferative GN sind in 0 Tab. 2 zusammengefasst. 
Alle Patienten mit hepatitisassoziierten Glomerulonephritiden sollten eine symptomatische antihypertensive und antiproteinurische Therapie erhalten (ACEHemmer oder $\mathrm{AT}_{2}$-Rezeptorblocker, Diuretika). Im Übrigen sollten die Komplikationen eines allfälligen nephrotischen Syndroms (Hyperlipidämie, Hyperkoagulabilität, Hypogammaglobulinämie) wie bei den entsprechenden primären Formen behandelt werden.

Für die $H B V$-assoziierte membranöse $G N$ kann IFNa als First-line-Therapie empfohlen werden. Bei Kontraindikation oder Unverträglichkeit steht Lamivudin zur Verfügung mit möglicherweise etwas geringerer Wirksamkeit. Für neuere Substanzen liegen keine Daten vor. Von einer unspezifischen Immunsuppression wird abgeraten. Wenn diese jedoch unvermeidlich erscheint, sollte sie nur in Kombination mit einer antiviralen Therapie eingesetzt werden. Die Prognose der HBV-assoziierten membranösen GN ist bei erfolgreicher Suppression der Virusreplikation günstig.

Als First-line-Therapie der HCV-assoziierten kryoglobulinämischen membranoproliferativen $G N$ in milden Fällen sollte eine antivirale Therapie mit IFNa und Ribavirin eingeleitet werden. Bei schwerem Verlauf mit rasch fortschreitendem Nierenversagen, schwerem nephrotischem Syndrom oder systemischer Vaskulitis kann eine immunsuppressive Therapie mit Plasmapherese, Prednison und eventuell Rituximab eingesetzt werden, gefolgt von einer antiviralen Therapie. Für Patienten mit Kontraindikation (z. B. Status nach Nierentransplantation) oder Unverträglichkeit von IFNa gibt es mit Rituximab nun eine neue alternative Therapie der Glomerulonephritis, die jedoch keinen Einfluss auf die HCV-Replikation und die HCV-assoziierte Lebererkrankung hat.

\section{Korrespondierender Autor}

\section{PD Dr. T. Fehr}

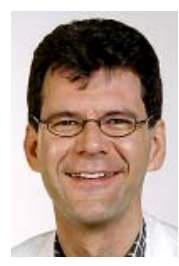

Klinik für Nephrologie, Universitätsspital

Rämistraße 100, 8091 Zürich, Schweiz

thomas.fehr@usz.ch
Interessenkonflikt. Es besteht kein Interessenkonflikt. Der korrespondierende Autor versichert, dass keine Verbindung mit einer Firma, deren Produkt in dem Artikel genannt ist, oder einer Firma, die ein Konkurrenzprodukt vertreibt, bestehen. Die Präsentation des Themas ist unabhängig und die Darstellung der Inhalte produktneutral.

\section{Literatur}

1. Agnello V, Abel G (1997). Localization of hepatitis $C$ virus in cutaneous vasculitic lesions in patients with type II cryoglobulinemia. Arthritis Rheum 40 (11): 2007-2015

2. Alric L, Plaisier E, Thebault S et al. (2004) Influence of antiviral therapy in hepatitis $C$ virus-associated cryoglobulinemic MPGN. Am J Kidney Dis 43 (4): 617-623

3. Amarapurkar DN, Amarapurkar AD (2002) Extrahepatic manifestations of viral hepatitis. Ann Hepatol 1 (4): 192-195

4. Basse G, Ribes D, Kamar N et al. (2006) Rituximab therapy for mixed cryoglobulinemia in seven renal transplant patients. Transplant Proc 38 (7): 2308 2310

5. Bhimma R, Coovadia HM (2004) Hepatitis B virus-associated nephropathy. Am J Nephrol 24 (2): 198-211

6. Bhimma R, Coovadia HM, Kramvis A et al. (2002) Treatment of hepatitis $B$ virus-associated nephropathy in black children. Pediatr Nephrol 17 (6): 393-399

7. Blackard JT, Kemmer N, Sherman KE (2006) Extrahepatic replication of HCV: insights into clinical manifestations and biological consequences. Hepatology 44 (1): 15-22

8. Bruchfeld A, Lindahl K, Stahle L et al. (2003) Interferon and ribavirin treatment in patients with hepatitis $\mathrm{C}$-associated renal disease and renal insufficiency. Nephrol Dial Transplant 18 (8): 1573-1580

9. Cacoub P, Saadoun D, Bourliere M et al. (2005) Hepatitis $B$ virus genotypes and extrahepatic manifestations. J Hepatol 43 (5): 764-770

10. D'Amico G (1998) Renal involvement in hepatitis C infection: cryoglobulinemic glomerulonephritis. Kidney Int 54 (2): 650-671

11. Fabrizi F, Colucci P, Ponticelli C, Locatelli F (2002) Kidney and liver involvement in cryoglobulinemia. Semin Nephrol 22 (4): 309-318

12. Fehr T, Bachmann MF, Bucher E et al. (1997) Role of repetitive antigen patterns for induction of antibodies against antibodies. J Exp Med 185 (10): 17851792

13. Germuth FG, Jr., Rodriguez E, Lorelle CA et al. (1979) Passive immune complex glomerulonephritis in mice: models for various lesions found in human disease. II. Low avidity complexes and diffuse proliferative glomerulonephritis with subepithelial deposits. Lab Invest 41 (4): 366-371

14. Gilbert RD, Wiggelinkhuizen J (1994) The clinical course of hepatitis B virus-associated nephropathy. Pediatr Nephrol 8 (1): 11-14

15. Guillevin L, Mahr A, Callard P et al. (2005) Hepatitis $B$ virus-associated polyarteritis nodosa: clinical characteristics, outcome, and impact of treatment in 115 patients. Medicine (Baltimore) 84 (5): 313322

16. He XY, Fang LJ, Zhang YE et al. (1998) In situ hybridization of hepatitis B DNA in hepatitis B-associated glomerulonephritis. Pediatr Nephrol 12 (2): 117-120
17. Johnson RJ, Gretch DR, Couser WG et al. (1994) Hepatitis $C$ virus-associated glomerulonephritis. Effect of alpha-interferon therapy. Kidney Int 46 (6): 1700-1704

18. Kamar N, Rostaing L, Alric L (2006) Treatment of hepatitis C-virus-related glomerulonephritis. Kidney Int 69 (3): 436-439

19. Lai KN, Tam JS, Lin HJ, Lai FM (1990) The therapeutic dilemma of the usage of corticosteroid in patients with membranous nephropathy and persistent hepatitis $B$ virus surface antigenaemia. $\mathrm{Ne}$ phron 54 (1): 12-17

20. Lin CY (1995) Treatment of hepatitis B virus-associated membranous nephropathy with recombinant alpha-interferon. Kidney Int 47 (1): 225-230

21. Medina J, Garcia-Buey L, Moreno-Otero R (2004) Hepatitis $C$ virus-related extra-hepatic disease--aetiopathogenesis and management. Aliment Pharmacol Ther 20 (2): 129-141

22. Meyers CM, Seeff LB, Stehman-Breen CO, Hoofnagle JH (2003) Hepatitis C and renal disease: an update. Am J Kidney Dis 42 (4): 631-657

23. Misiani R, Bellavita P, Fenili D et al. (1994) Interferon alfa-2a therapy in cryoglobulinemia associated with hepatitis C virus. N Engl J Med 330 (11): 751756

24. Palekar NA, Harrison SA (2005) Extrahepatic manifestations of hepatitis C. South Med J 98 (10): 1019-1023

25. Quartuccio L, Soardo G, Romano G et al. (2006) Rituximab treatment for glomerulonephritis in HCVassociated mixed cryoglobulinaemia: efficacy and safety in the absence of steroids. Rheumatology (Oxford) 45 (7): 842-846

26. Roccatello D, Baldovino S, Rossi D et al. (2004) Long-term effects of anti-CD20 monoclonal antibody treatment of cryoglobulinaemic glomerulonephritis. Nephrol Dial Transplant 19 (12): 30543061

27. Rossi P, Bertani T, Baio P et al. (2003) Hepatitis C virus-related cryoglobulinemic glomerulonephritis: long-term remission after antiviral therapy. Kidney Int 63 (6): 2236-2241

28. Sasso EH (2000) The rheumatoid factor response in the etiology of mixed cryoglobulins associated with hepatitis $C$ virus infection. Ann Med Interne (Paris) 151 (1): 30-40

29. Sayarlioglu H, Erkoc R, Dogan E et al. (2005) Mycophenolate mofetil use in hepatitis $B$ associatedmembranous and membranoproliferative glomerulonephritis induces viral replication. Ann Pharmacother 39 (3): 573

30. Szymanski IO, Pullman JM, Underwood JM (1994) Electron microscopic and immunochemical studies in a patient with hepatitis $C$ virus infection and mixed cryoglobulinemia type II. Am J Clin Pathol 102 (3): 278-283

31. Tang S, Lai FM, Lui YH et al. (2005) Lamivudine in hepatitis B-associated membranous nephropathy. Kidney Int 68 (4): 1750-1758

32. Zaja F, De Vita S, Mazzaro C et al. (2003) Efficacy and safety of rituximab in type II mixed cryoglobulinemia. Blood 101 (10): 3827-3834 
Hier steht eine Anze

橧 Springer 